\title{
Coda-wave Interferometry Analysis of Time-lapse VSP Data for Monitoring Geological Carbon Sequestration
}

Authors:

$$
\begin{gathered}
\text { Rongmao Zhou }{ }^{(1)} \text {, Lianjie Huang }{ }^{(1)} \text {, James T. Rutledge }{ }^{(1)} \\
\text { Michael Fehler }{ }^{(2)}
\end{gathered}
$$

Thomas M. Daley ${ }^{(3)}$ and Ernest L. Majer ${ }^{(3)}$

\section{Affiliation:}

(1) Geophysics Group, Los Alamos National Laboratory, Los Alamos, NM 87545

(2) Massachusetts Institute of Technology

(3) Lawrence Berkeley National Laboratory, Berkeley, CA 94720

\section{Address:}

\section{Email:}

(1) Geophysics Group, MS D443

Los Alamos National Laboratory

Los Alamos, NM 87545

(2) Department of Earth, Atmospheric, \& Planetary Sciences Massachusetts Institute of Technology

77 Massachusetts Avenue, 54-524

Cambridge, MA 02139

(3) Geophysics Department, MS 90-1116

Lawrence Berkeley National Laboratory

Berkeley, CA 94720

$$
\begin{aligned}
& \text { rongmaozhou@gmail.com (R. Zhou) } \\
& \text { ljh@lanl.gov (L. Huang) } \\
& \text { jrutledge@lanl.gov (J. Rutledge) } \\
& \text { fehler@mit.edu (Michael Fehler) } \\
& \text { tmdaley@lbl.gov (Thomas Daley) } \\
& \text { elmajer@lbl.gov (Ernest Majer) }
\end{aligned}
$$

Fax: $\quad 1-505-667-8487$ 


\section{Coda-wave Interferometry Analysis of Time-lapse VSP Data for Monitoring Geological Carbon Sequestration}

\section{ABSTRACT}

Injection and movement/saturation of carbon dioxide $\left(\mathrm{CO}_{2}\right)$ in a geological formation will cause changes in seismic velocities. We investigate the capability of coda-wave interferometry technique for estimating $\mathrm{CO}_{2}$-induced seismic velocity changes using time-lapse synthetic vertical seismic profiling (VSP) data and the field VSP datasets acquired for monitoring of injected $\mathrm{CO}_{2}$ in a brine aquifer in Frio, Texas, USA. Synthetic VSP data are calculated using a finite-difference elastic-wave equation scheme and a layered model based on the elastic Marmousi model. A possible leakage scenario is simulated by introducing seismic velocity changes in a layer above the $\mathrm{CO}_{2}$ injection layer. We find that the leakage can be detected by the detection of a difference in seismograms recorded after the injection compared to those recorded before the injection at an earlier time in the seismogram than would be expected if there was no leakage. The estimated mean velocity changes, from both synthetic and field VSP data, increase significantly for receiver positions approaching the top of a $\mathrm{CO}_{2}$ reservoir. Our results from field data suggest that the velocity changes caused by $\mathrm{CO}_{2}$ injection could be more than $10 \%$ and are consistent with results from a crosswell tomogram study. This study demonstrates that timelapse VSP with coda-wave interferometry analysis can reliably and effectively monitor geological carbon sequestration.

\section{KEY WORDS}


Coda-Wave Interferometry, Geologic Carbon Sequestration, Time-lapse VSP Monitoring, Vertical Seismic Profiling

\section{INTRODUCTION}

The goal of geological carbon sequestration is to permanently store large quantities of carbon dioxide $\left(\mathrm{CO}_{2}\right)$ within underground rock formations. It will be required to know with a high level of confidence that the injected $\mathrm{CO}_{2}$ remains sequestered permanently. Seismic monitoring could play an important role to monitor and ensure safe, effective storage of $\mathrm{CO}_{2}$. Time-lapse seismic reservoir monitoring is a process to acquire and analyze multiple seismic surveys, repeated at the same site over time, in order to image fluid-flow effects in a producing reservoir. As fluid saturations and pressures in the reservoir change, the seismic reflection properties change accordingly (Lumley, 2001). Time-lapse seismic monitoring provides information about changes in temperature, pressure, and volume change in fluid property within reservoir pore spaces at two different calendar dates. Time-lapse imaging has been successfully applied by the oil industry for reservoir monitoring (Santos and Harris, 2007). The ability to monitor reservoir changes as a function of time by the use of seismic methods can lead to better location of production and infill wells and the possibility of locating unswept zones (Ross et al., 1996).

A similar theoretical background can be applied to monitor geologic $\mathrm{CO}_{2}$ sequestration and to detect possible leakage. Depleted oil reservoirs, saline aquifers, and un-mineable coal seams have been suggested as potential storage sites. $\mathrm{CO}_{2}$ could permanently be stored within the rock pore spaces where oil/natural gas/water have been held for millions of years. Injection and movement/saturation of $\mathrm{CO}_{2}$ in a geological formation will cause changes in seismic velocities 
and attenuation, which result in changes in seismic-wave scattering and propagation. The changes in seismic velocities are believed to be associated jointly with changes in fluid saturation $\left(\mathrm{CO}_{2}\right.$ replacing water and oil) and in pressure (increase in pore pressure due to the injection process) (Harris et al., 1996). Wang et al. (1998) investigated the effect of $\mathrm{CO}_{2}$ floods on the seismic velocities in a carbonate rock (dolostone). They found that compressional-wave (Pwave) velocity $\mathrm{V}_{\mathrm{P}}$ decreases from a minimum $3.0 \%$ to as high as $10.9 \%$, while shear-wave (Swave) velocity Vs decreases from $3.3 \%$ to $9.5 \%$ as the reservoir rocks are flooded with $\mathrm{CO}_{2}$ under in-situ conditions. Their results show that the combined effects of pore pressure buildup and fluid substitution caused by $\mathrm{CO}_{2}$ flooding make it feasible to monitor the $\mathrm{CO}_{2}$ flood process and to map the flooded zones seismically. Rock and fluid physics measurements and modeling suggest that $\mathrm{CO}_{2}$ can cause a $4-6 \%$ decrease in $\mathrm{V}_{\mathrm{P}}$ with a corresponding $15-20 \%$ change in reflection amplitude. Time-lapse surface seismic and vertical seismic profiling (VSP) surveys for monitoring $\mathrm{CO}_{2}$ sequestration also showed changes in $\mathrm{P}$-wave and $\mathrm{S}$-wave velocities due to $\mathrm{CO}_{2}$ injection and strong reflections from injection regions (Arts et al., 2004; Daley et al., 2007). Time-lapse crosswell tomograms show P-wave reductions of more than $10 \%$ in some formations within the reservoir zone and some changes are as large as 20\% (Harris et al., 1996, Daley et al, 2007).

The usefulness of temporal changes, however, is limited by the accuracy and precision with which velocity measurements can be made (Poupinet et al., 1984; Roberts et al., 1992, Sneider et al., 2002). Poupinet et al., (1984) introduced the coda-wave interferometry method for estimating nonlinear behavior in seismic velocity. It has been applied to different studies, such as probing the relative location of seismic sources (Snieder and Vrijlandt, 2005), monitoring of rapid 
temporal change in a volcano (Grêt et al., 2005), and time-lapse monitoring of rock properties in a laboratory environment (Grêt et al., 2006). The coda-wave interferometry method can be used to detect spatially localized changes using single scattering (Pacheco and Snieder, 2006) and multiple scattering (Pacheco and Snieder, 2005).

The purpose of this study is to explore the capability of the coda-wave interferometry method for monitoring geologic carbon sequestration. We use it to estimate seismic velocity changes due to $\mathrm{CO}_{2}$ injection using synthetic and field time-lapse VSP data. The coda waves in upgoing VSP data are waves that have been multiply scattered from geologic layers below the receiver positions. First, we will briefly outline the coda-wave interferometry methodology, and using repeating earthquake events to validate the algorithm and explore the effects of temporal windows on the results of estimation. Then, we will apply the coda-wave interferometry methodology to synthetic time-lapse VSP data for monitoring $\mathrm{CO}_{2}$ injection, and investigate the monitoring of the possible $\mathrm{CO}_{2}$ leakage scenario. We will also apply the coda-wave interferometry method to the field time-lapse VSP datasets acquired for monitoring $\mathrm{CO}_{2}$ injection into a brine aquifer in Frio, Texas, USA.

\section{METHODOLOGY AND VALIDATION OF THE ALGORITHM}

Poupinet et al., (1984) first proposed to measure small changes in coda waves to infer small changes in velocity of a region. Later, Snieder et al. (2002) proposed a method termed "Coda Wave Interferometry" for detecting the presence of temporal changes in the medium. Here we 
briefly outline the coda-wave interferometry technique; more detailed information can be found in Snieder et al. (2002). Suppose that a strongly scattering medium is excited by a repeatable source, and that the medium changes with time. Before the change in the medium occurs, the unperturbed wave field $u^{(u)}(t)$ can be written as

$$
u^{(u)}(t)=\sum_{T} A_{T}(t)
$$

which is a sum of waves propagating along the multiple scattering trajectories $T$ in the medium, where $t$ denotes time and $A_{T}(t)$ is the wave propagating along trajectory $T$. When the medium velocity changes over time, the dominant effect is a change $\tau_{T}$ in the arrival times of the waves that propagate along different trajectory $T$, so that the perturbed wave field is given by

$$
u^{(p)}(t)=\sum_{T} A_{T}\left(t-\tau_{T}\right)
$$

The change in the waveforms can be quantified by computing the time-shifted cross-correlation over a time window at center time $t$ with temporal width $2 t_{w}$ :

$$
R\left(t_{S}\right) \equiv \frac{\int_{t-t_{w}}^{t+t_{w}} u^{(u)}\left(t^{\prime}\right) u^{(p)}\left(t^{\prime}+t_{S}\right) d t^{\prime}}{\left(\int_{t-t_{w}}^{t+t_{w}}\left(u^{(u)}\left(t^{\prime}\right)\right)^{2} d t^{\prime} \int_{t-t_{w}}^{t+t_{w}}\left(u^{(p)}\left(t^{\prime}\right)\right)^{2} d t^{\prime}\right)^{1 / 2}}
$$

where $t_{S}$ is the time shift of the perturbed waveform relative to the unperturbed one. When the waves are not perturbed, $u^{(p)}(t)=u^{(u)}(t)$, and the time-shifted cross-correlation is equal to unity for a zero lag time $R\left(t_{S}=0\right)=1$. When the perturbed wave within the measurement time window is a time-shifted version of the original wave, $u^{(p)}(t)=u^{(u)}(t-\tau)$, and $R\left(t_{S}\right)$ attain its maximum at $t_{S}=\tau$. In general, the time-shifted cross-correlation $R\left(t_{S}\right)$ attains its maximum at 
a time $t_{S}=t_{\max }$ when

$$
t_{\max }=\tau
$$

The shift time is given by the average perturbation of the travel time of the waves that arrive in the employed time window. For each time interval, the time shift between the perturbed and unperturbed waves is determined by computing the time-shifted cross-correlation in equation (3) and by picking the time for which the cross-correlation coefficient attains its maximum at $t_{\max }$. The relative velocity change for each time interval is the given by

$$
\frac{\delta v}{v}=-\frac{t_{\max }}{t}=-\frac{\tau}{t}
$$

This velocity change is a function of the center time $t$ of the employed time windows.

For spatially localized changes, the average or mean travel time change is given the by (Pacheco and Snieder, 2005):

$$
\langle\tau(t)\rangle=-\int_{V} K\left(r^{\prime}, t\right) \frac{\delta v\left(r^{\prime}\right)}{v\left(r^{\prime}\right)} d V\left(r^{\prime}\right)
$$

where $\langle\tau(t)\rangle$ is the mean travel time change of the multiply scattering waves at travel time $t$ due to the spatially localized velocity change $\frac{\delta v\left(r^{\prime}\right)}{v\left(r^{\prime}\right)}$ within the volume $V$, and $K\left(r^{\prime}, t\right)$ is the integration kernel satisfying

$$
t=\int_{V} K\left(r^{\prime}, t\right) d V\left(r^{\prime}\right) .
$$


We define the mean velocity change $\left\langle\frac{\delta v}{v}\right\rangle$ as

$$
\left\langle\frac{\delta v}{v}\right\rangle \equiv \frac{\int_{V} K\left(r^{\prime}, t\right) \frac{\delta v\left(r^{\prime}\right)}{v\left(r^{\prime}\right)} d V\left(r^{\prime}\right)}{\int_{V} K\left(r^{\prime}, t\right) d V\left(r^{\prime}\right)}=-\frac{\langle\tau(t)\rangle}{t} .
$$

We use the mean velocity change $\left\langle\frac{\delta v}{v}\right\rangle$ for qualitative detection of reservoir changes due to $\mathrm{CO}_{2}$ injection.

We implement the coda-wave interferometry algorithm in Matlab environment. To test the codes and explore the effects of parameters, such as temporal window length, on the results of velocity change estimations, we apply the coda-wave interferometry method to repeating earthquake events. The testing results indicate that a shorter window length (about 1 3 wave periods) have some fluctuations and include outliers at some center times. When the window length is greater than 4 wave periods, the results of the coda-wave interferometry are almost the same. The results validate our computer codes of the coda-wave interferometry method and show that the technique could be used to estimate the relative changes using seismic data with longer window lengths.

\section{APPLICATION TO SYNTHETIC TIME-LAPSE VSP DATA}

We first study the feasibility of the coda-wave interferometry method for monitoring geologic carbon sequestration using synthetic time-lapse VSP data. We also investigate whether this 
technique could be used to detect the possible leakage scenario from the $\mathrm{CO}_{2}$ injection, which is one of main tasks of monitoring geologic $\mathrm{CO}_{2}$ sequestration. We use layered elastic models $(\mathrm{P}-$ wave velocity: $\mathrm{V}_{\mathrm{P}}$; $\mathrm{S}$-wave velocity: $\mathrm{V}_{\mathrm{S}}$; and density: $\rho$ ) to calculate synthetic VSP seismograms (Fig. 1). The reference layered model is based on a portion of the elastic Marmousi model (Martin et al., 2006) without the top water layers (Fig. 1). The VSP source and receiver geometries for the synthetic seismogram calculations are depicted in Fig. 1. The red star denotes the source; red triangles are receivers; and the green filled rectangle represents the $\mathrm{CO}_{2}$ injection layer with a thickness of $200 \mathrm{~m}$. The VSP offset is $1000 \mathrm{~m}$ and the source is located $50 \mathrm{~m}$ below the surface. The synthetic VSP data calculated using the reference model are used as the preinjection, baseline VSP data. To obtained post-injection synthetic VSP data, we change the velocities values in the $\mathrm{CO}_{2}$ injection layer (the green layer in Fig. 1) and the resulting model is called Test Model 1. Rock and fluid physics measurements and modeling suggest that $\mathrm{CO}_{2}$ can cause a 4-6\% decrease in P-wave velocity and a 5-10\% decrease in S-wave velocity (Davis et al., 2003). Test Model 1 has 6\% decrease in P-wave velocity and 5\% decrease in S-wave velocity relative to the reference model. To simulate a possible leakage scenario, Test Model 2 is created by changing model parameters in a layer (cyan) above the $\mathrm{CO}_{2}$ injecting layer (green). Test Model 2 for the leakage scenario contains an additional leakage layer with 3\% P-wave velocity decrease and 3\% S-wave velocity decrease relative to Test Model 1.

A finite-difference elastic-wave-equation scheme (Cheng, 1994; Kamm et al., 1996) is used to calculate synthetic seismograms for an explosive point source with a center frequency of $25 \mathrm{~Hz} . \mathrm{P}$ and $\mathrm{S}$ waves were separated using divergence and curl of the wavefield (Sun and McMechan, 2001). Upgoing and downgoing wavefield separation is accomplished in the frequency- 
wavenumber $(f-k)$ domain using the technique of contour-slice filtering (Suprajitno and Greenhalgh, 1985; Hardage, 2000). Since most of the downgoing wavefield does not pass through the injection layer, we only use upgoing waves for our monitoring studies. Figure 2 shows the upgoing waves of synthetic seismograms for the reference model (upper-left) and the Test Model 1 (upper-right). The upgoing waves for the Test Model 1 at receiver \#1, \#201, \#401, $\# 601, \# 801$ and \#1001 superposed with the waveforms from the reference model are also presented in Figure 2. The velocity change estimated using the coda-wave interferometry technique for time-lapse VSP data is the velocity change over the entire travel path. We calculate the temporal velocity changes at the centers of a moving time window using the coda-wave interferometry method, and then obtain the mean velocity change by averaging the temporal velocity changes over entire analyzed time record. Figure 3 presents the comparison of results for Test Model 1 relative to the reference model (blue) and Test Model 2 (leakage scenario) relative to the reference model (red) at receivers \#1, \#201, \#401, \#601, \#801 and \#1001 as marked as solid blue triangles in Fig. 1. The estimated temporal velocity changes occur earlier for Test Model 2 compared to those for Test Model 1 at all receivers. The earlier occurrences of changes imply some velocity changes occurred in the upper layers over the injection layer, indicating $\mathrm{CO}_{2}$ leakage.

For each receiver, we estimate the velocity change versus the time as the examples shown in Figure 3, then we average them over whole time window to obtain the mean velocity change for each receiver. Figure 4 shows the mean P-wave velocity change for each receiver versus the receiver depth with temporal window length of 5 periods for the central frequency; and Figure 5 presents estimated mean velocity changes of the S-wave. The mean velocity change increases 
significantly when receivers approach the top of the $\mathrm{CO}_{2}$ injection layers. The results for the leakage scenario show the overall contribution from the injection and leakage layers.

At each receiver position, the upgoing waves have propagated varying distances through the section, of which only a portion corresponds to the $200 \mathrm{~m}$ injection interval where the velocity change occurs. Therefore, the receivers with the maximum P-wave velocity changes are those near the injection layer since the $200 \mathrm{~m}$ interval makes up a larger fraction of the total travel paths over which change is being measured. The receivers with the maximum estimated P-wave velocity changes are those near the injection layer. For these receivers, the total lengths of wave propagation paths are around $2000 \mathrm{~m}$, and the propagation paths within the injection layer are approximately $400 \mathrm{~m}$, which are nearly $20 \%$ of the entire wavepaths. The maximum mean velocity changes for these receivers are approximate $0.6 \%$, roughly $10 \%$ of the given changes in the models. After taking in account the effect of the propagation paths through the injection layer over the total paths, the estimated mean velocity changes are half of the input value. The maximum estimated changes from Test Model 2 are approximately 1.5 times bigger than results from Test Model 1, which is the same as given velocity changes ratios for both models relative to the reference model. These results reveal characteristics of the coda-wave interferometry as a detector of the relative temporal changes.

\section{APPLICATION TO FIELD TIME-LAPSE VPS DATA}

$\mathrm{CO}_{2}$ Injection Test in Frio, Texas 
The field time-lapse VSP data were collected in Frio for a small scale (1,600 ton) pilot test of $\mathrm{CO}_{2}$ injection into a brine aquifer. The goals of the pilot study were to safely inject $\mathrm{CO}_{2}$, model the expected $\mathrm{CO}_{2}$ flow, sample the fluid in an up-dip observation well, and monitor the resulting $\mathrm{CO}_{2}$ plume (Hovorka et al. 2006, Doughty et al. 2007). The selected aquifer is a part of the on-shore Gulf of Mexico Frio formation sandstone. The experimental site is in an oil field and an idle well was used as an observation well. A new well was drilled for injection about $30 \mathrm{~m}$ offset from the existing observation well.

Time-lapse VSP data were acquired using a tubing-deployed, 80-level, 3-component geophone string and explosive sources. The explosive shot holes were roughly $18.3 \mathrm{~m}(60 \mathrm{ft})$ deep and were located $130 \mathrm{~m}$ to $1500 \mathrm{~m}$ away from the instrumented injection well on multiple azimuths. Each shot contained $1.6 \mathrm{~kg}(3.5 \mathrm{lbs})$ of dynamite. The data analyzed here are from the source offset $130 \mathrm{~m}$ updip (north) from the injection well, This is the azimuth for which the propagation is most likely 2D (in the plane of source and sensors). The time-lapse VSP data were acquired in July 2004 (pre-injection survey) and in late November 2004 (post-injection surveys), which was 1.5 months after the $\mathrm{CO}_{2}$ was injected into the upper $\mathrm{C}$-sand of the Frio Formation at depth from 1,528.5 m to $1,534.7 \mathrm{~m}$ (Daley et al., 2007).

Daley et al. (2007) described the steps of data processing for these dataset. After applying some standard VSP processing steps, that included frequency-wavenumber separation of downgoing and upgoing wavefield, and converting reflections to two-way travel time, the amplitudes were equalized using reflections from an interface above the reservoir as a reference. This equalization assumes that amplitude changes in reflectors above the injection interval are due to shallow subsurface changes (such as soil moisture saturation) or changes in the seismic source amplitude. 
Therefore, the amplitude change measured in the shallow reflector is subtracted from all the data. Figure 6 presents the pre- and post-injection up-going VSP data after these pre-process procedures from offset 1 , which illustrates the high data quality.

\section{Coda-wave interferometry analysis}

The coda-wave interferometry method is applied to these pre- and post-injection VSP data to estimate seismic velocity changes caused by the $\mathrm{CO}_{2}$ injection. The central frequency for these data is approximately $30 \mathrm{~Hz}$. For the coda-wave interferometry analyses, we use a temporal window length of 0.198 and $0.297 \mathrm{sec}$, which is equivalent to 6 and 9 periods at the center frequency, respectively. The results are similar for both cases. Figure 7 presents the mean velocity changes versus the depth of VSP receivers with a time window length of 6 periods. The

mean velocity change is nearly constant at geophones above $1400 \mathrm{~m}$; increases significantly at receiver positions near the top of the $\mathrm{CO}_{2}$ injection layer; and then decreases with increasing receiver depth below the injection layer.

Figure 7 shows that the maximum estimated velocity change is about $0.045 \%$. The thickness of the injection layer was about $6.2 \mathrm{~m}$ (Daley et al., 2007). By taking into account the relative ray path through the injection layer to the entire path of the upgoing waves, the actual average velocity change could be between $10 \%$ and $20 \%$.

To verify the estimated velocity changes from the time-lapse VSP data, a genetic algorithm and ray tracing method are used to invert the velocity model from the picked first break times. The left panel of Fig. 8 shows a comparison of the picked travel times (red) and the calculated travel 
times from one of the best inverted models on the right. We also compare the inverted velocity structure with the $\log$ data. The velocities below $1100 \mathrm{~m}$ fit well with the log data and the firstbreak time fit well for VSP receiver at all depth ranges. This inverted Vp model is used to calculate pre-injection VSP synthetic seismograms. Vs model is obtained from Vp according to the $\mathrm{Vp} / \mathrm{Vs}$ ratio obtained from log data. The results from the cross-well tomogram study show seismic P-wave velocity decrease up to $500 \mathrm{~m} / \mathrm{s}$ (Daley et al., 2007) in the injection region. We change velocities in the injection layer by decreasing Vp by $200 \mathrm{~m} / \mathrm{s}$ and Vs by $50 \mathrm{~m} / \mathrm{s}$, and use this modified model to calculate the post-injection VSP synthetic data. The synthetic VSP data are calculated using a finite-difference elastic-wave-equation scheme (Cheng, 1994; Kamm et al., 1996) with an explosive source that has a center frequency of $30 \mathrm{~Hz}$ and is located $130 \mathrm{~m}$ away from the monitoring well (a similar geometry as the Frio field experiment).

Figure 9 is the comparison of estimated velocity changes from synthetic time-lapse (red) and field VSP (blue). Both estimated velocity changes reach a maximum near the injection layer with a value of approximately $0.045 \%$. The results from time-lapse filed VSP data are consistent with those from synthetic VSP data with the mean P-wave velocity changes $200 \mathrm{~m} / \mathrm{sec}$ in the injection layer.

\section{CONCLUSIONS}

We have investigated the feasibility of the coda-wave interferometry analysis for monitoring $\mathrm{CO}_{2}$ injection and detecting a $\mathrm{CO}_{2}$ leakage scenario using time-lapse VSP data. The coda-wave interferometry method can accurately determine the time in the seismograms when the temporal velocity change occurs for a given leakage scenario. If the center time of the first temporal velocity change shifts to earlier 
portions of time-lapse seismograms, it indicates that some $\mathrm{CO}_{2}$ could have leaked to the upper layers. This provides a quick and reliable tool for detecting $\mathrm{CO}_{2}$ leakage using time-lapse VSP data. Synthetic study results suggest that the estimated relative temporal change is equivalent to the given changes after we take into account the ratio of the length of the wave path through the injection layer where velocity changes occur over the length of the entire propagation path. We have applied coda-wave interferometry to time-lapse field VSP data from the Frio project. The results from the field data indicate that the mean velocity changes caused by injecting $\mathrm{CO}_{2}$ into the Frio Formation could be larger than $10 \%$. This result is consistent with results from the time-lapse crosswell tomography study for the same field experiment. Our studies with synthetic and field time-lapse VSP data suggest that coda-wave interferometry analysis could be a reliable and effective tool for monitoring geological carbon sequestration.

\section{ACKNOWLEDGEMENTS}

This work was supported by the U.S. Department of Energy through contract DE-AC52-06NA25396 to Los Alamos National Laboratory. Field VSP data were collected by Lawrence Berkeley National Laboratory for the GEOSEQ project for the Assistant Secretary for Fossil Energy, Office of Coal and Power Systems through the National Energy Technology Laboratory, of the U.S. Department of Energy, under contract No. DE-AC02-05CH11231.

\section{REFERENCES}

Arts, R., O. Eiken, A. Chadwick, P. Zweigel, L. van der Meer, B. Zinszner, 2004. Monitoring of $\mathrm{CO}_{2}$ injected at Sleipner using time-lapse seismic data. Energy 29, 1383-1392.

Cheng, N., 1994. Borehole wave propagation in isotropic and anisotropic media: threedimension finite difference approach, Ph.D. Thesis, Massachusetts Institute of 
Technology.

Davis, T. L., M. J. Terrell, R. D. Benson, R. Cardona, R. R. Kendall, R. Winarsky, 2003. Multicomponent seismic characterization and monitoring of $\mathrm{CO}_{2}$ flood at Weyburn Field, Saskatchewan. The Leading Edge 22, 696-697.

Daley, T.M, L.R. Myer, E.L. Majer, J.E. Peterson, 2005. Acquisition of time-lapse, 6component, P- and S-wave, crosswell seismic survey with orbital vibrator, and of timelapse VSP for CO2 injection monitoring, $75^{\text {th }}$ Ann. Internat. Mtg., Soc. Expl. Geophys., Expanded Abstracts, 1277-1279.

Daley, T. M., L. R. Myer, J. E. Peterson, E. L. Majer and G. M. Hoversten, 2007. Time-lapse crosswell seismic and VSP monitoring of injected $\mathrm{CO}_{2}$ in a brine aquifer, Environ. Geol. DOI 10.1007/s00254-007-0943-z.

Doughty, C., B. M. Freifeld, R. C. Trautz, 2007. Site characterization for $\mathrm{CO}_{2}$ geologic storage and vice versa: the Frio brine pilot, Texas, USA as a case study, Environ. Geol. DOI 101007/s00254-007-0942-0.

Grêt, A., R. Snieder, R. C. Aster, P. R. Kyle, 2005. Monitoring rapid temporal change in a volcano with coda wave interferometry, Geophys. Res. Lett. 32, L06304, doi:10.1029/2004GL021143.

Grêt, A., R. Snieder, J. Scales, 2006. Time-lapse monitoring of rock properties with coda wave interferometry, J. Geophys. Res. 111, B03305, doi: 10.1029/2004JB003354.

Harris, J. M., R., T. Langan, T. Fasnacht, D. Melton, B. Smith, J. Sinton, H. Tan, 1996. Experimental verification of seismic monitoring of $\mathrm{CO} 2$ injection in carbonate reservoirs, $66^{\text {th }}$ Ann. Internat Mtg., Soc. Expl. Geophys., Expended Abstracts, 1870-1872.

Hardage, B. A., 2000. Handbook of Geophysical Explosion, Vol. 14: Vertical Seismic Profiling: 
Principles, third updated and revised edition, Elsevier Science Ltd.

Hovorka, S D., S. M. Benson, C. Doughty, B. M. Freifeld, S. Sakuriai, T. M. Daley, Y. K.Kharaka, M. H. Holtz, R. C. Trautz, H. S. Nance, L. R. Myer, K.G. Knauss, 2006. Measuring permanence of $\mathrm{CO}_{2}$ storage in saline formations: the Frio experiment, Environ. Geosci. 13, No. 2, 105-121.

Kamm, J. R., R. J. Bos, E. M. Jones, 1996. User's Guide to AFD v. 1.0, Los Alamos National Laboratory, LA-UR-96-853.

Lumley, D. E., 2001. Time-lapse seismic reservoir monitoring, Geophysics 66, 50-53.

Martin, G. S., R. Wiley, K. J. Marfurt, 2006. Marmousi2: An elastic upgrade for Marmousi, The Leading Edge. 25, 156-166.

Pacheco, C. and R. Snieder, 2005. Time-lapse time time change of multiply scattered acoustic waves, J. Acoust. Soc. Am. 118, 1300-1310.

Pacheco, C. and R. Sneider, 2006. Time-lapse traveltime change of singly scattered acoustic waves, Geophy. J. Int. 165, 485-500.

Poupinet, G., W. L. Ellsworth, and J. Frechet, 1984. Monitoring velocity variations in the crust using earthquake doublets: an application to the Calaveras Fault, California, J. Geophy. Res. 89, 5719-5731.

Roberts, P. M., W. S. Phillips, M. C. Fehler, 1992. Development of the active doublet method for measuring small velocity and attenuation changes in solids, J. Acoust. Soc. Am. 91, 32913302.

Ross, C. P., G. B. Cunningham, D. P. Weber, 1996. Inside the crossequalization black box, The Leading edge 15, 1233-1240. 
Santos, E. T. F., J. M. Harris, 2007. Time-lapse diffraction tomography for trigonal meshes with temporal data integration applied to $\mathrm{CO}_{2}$ sequestration monitoring, $77^{\text {th }}$ Ann. Internat Mtg., Soc. Expl. Geophys, Expanded Abstracts, 2959-2963.

Snieder, R., A. Grêt, H. Douma, J. Scales, 2002. Coda wave interferometry for estimating nonlinear behavior in seismic velocity, Science 295, 2253-2255.

Snieder, R., M. Vrijlandt, 2005. Constraining the source separation with coda wave interferometry: Theory and application to earthquake doublets in the Hayward fault, California, J. Geophys. Res., 110, B04301, doi:10.1029/2004JB003317.

Sun, R. and G. A. McMechan, 2001. Scalar reverse-time depth migration of prestack elastic seismic data, Geophysics 66, 1519-1527.

Suprajitno, M., S. A. Greenhalgh, 1985. Separation of upgoing and downgoing waves in vertical seismic profiling by contour-slice filtering, Geophysics 50, 950-962.

Waldhauser, F., W. L. Ellsworth, 2002. Fault structure and mechanics of the Hayward Fault, California, from double-difference earthquake locations, J. Geophys. Res. 107, B3, 2054, 10.1029/2000JB000084.

Wang, Z., M. E. Cates, R. L. Langan, 1998. Seismic monitoring of $\mathrm{CO}_{2}$ flood in a carbonate reservoir: A rock physics study, Geophysics 63, 1604-1617. 


\section{Figures Captions:}

Figure 1. Left: The elastic Marmousi model (P-wave velocity:Vp; S-wave velocity: Vs and Density: $\rho$ ); Middle: A profile of the elastic Marmousi model at horizontal location 12000m (thin dotted lines on left) and a modified layered model (thick solid lines) used for synthetic seismogram calculations; Right: VSP geometry for synthetic seismogram calculations and a sketch of possible leakage scenario with parameters changes in the leakage layer (cyan) and the injection layer (green).

Figure 2. Synthetic seismograms of upgoing waves for the reference model (upper-left) and test model 1 (upper-right) at receiver \#50,\#100, \#150, \#200, ..., \#1000. Lower panel is the plot of the synthetic seismograms for the reference model (blue) superposing with synthetic seismograms for test model 1 (red) at receiver \#1, \#201, \#401, \#601, \#801, \#1001.

Figure 3. Temporal velocity changes estimated using the coda-wave interferometry technique from synthetic seismograms for test model 1 (solid blue line with crosses) and test model 2 (solid red with plus) relative to the reference model at receivers \#1, \#201, \#401, \#601, \#801, \#1001 (see Fig.1). The maximum correlation functions between the reference model and test model 1 (dotted blue with crosses), the reference model and test model 2 (dotted red with plus) at each moving window is ploted in each sub panel.

Figure 4. The estimated mean P-wave velocity change versus the receiver depth. The blue curve is for test model 1 , and the red one is for test model 2 relative to the reference model. The 
green layer is the $\mathrm{CO}_{2}$ injection layer, and the cyan layer is a leakage layer.

Figure 5. The estimated mean S-wave velocity change versus the receiver depth. The blue curve is for test model 1 , and the red one is for test model 2 relative to the reference model. The green layer is the $\mathrm{CO}_{2}$ injection layer, and the cyan layer is a leakage layer.

Figure 6. Vertical component VSP seismograms recorded from shot 1 (Left: Pre-Injection; and Right: Post-Injection). The injection layer is marked as thick green line at depth range of 1528.5 and $1534.7 \mathrm{~m}$.

Figure 7. The mean velocity change versus the receiver depth for the field time-lapse VSP data set. The thick green line indicates the $\mathrm{CO}_{2}$ injection layer.

Figure 8. Left: Comparison of picked (red) first break time and calculated (blue) first break time from the inverted model on the right; Right: One of best fitting models inverted using genetic algorithms. Thick green line denotes the injection layer; cyan and magenta curves are the p-wave, and s-wave velocity log data, respectively.

Figure 9. Comparison of estimated velocity changes vs. VSP receiver depth for field VSP data (blue) and synthetic time-lapse VSP data (red) with P-wave velocity reduction 200 $\mathrm{m} / \mathrm{sec}$ and $\mathrm{S}$-wave velocity reduction $50 \mathrm{~m} / \mathrm{sec}$. 

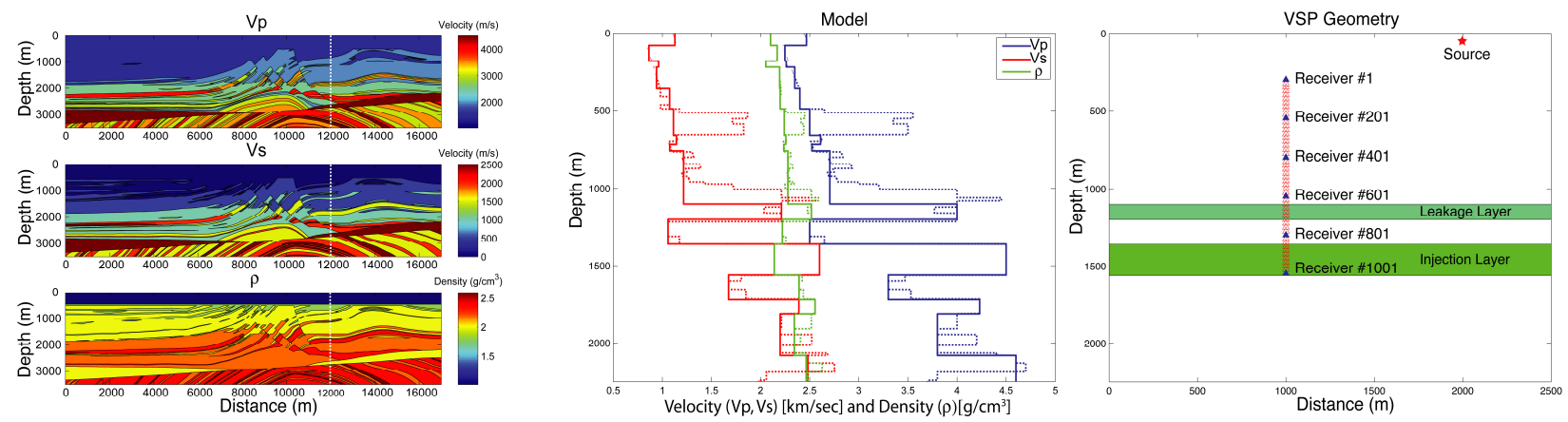

\section{Figure 1.}
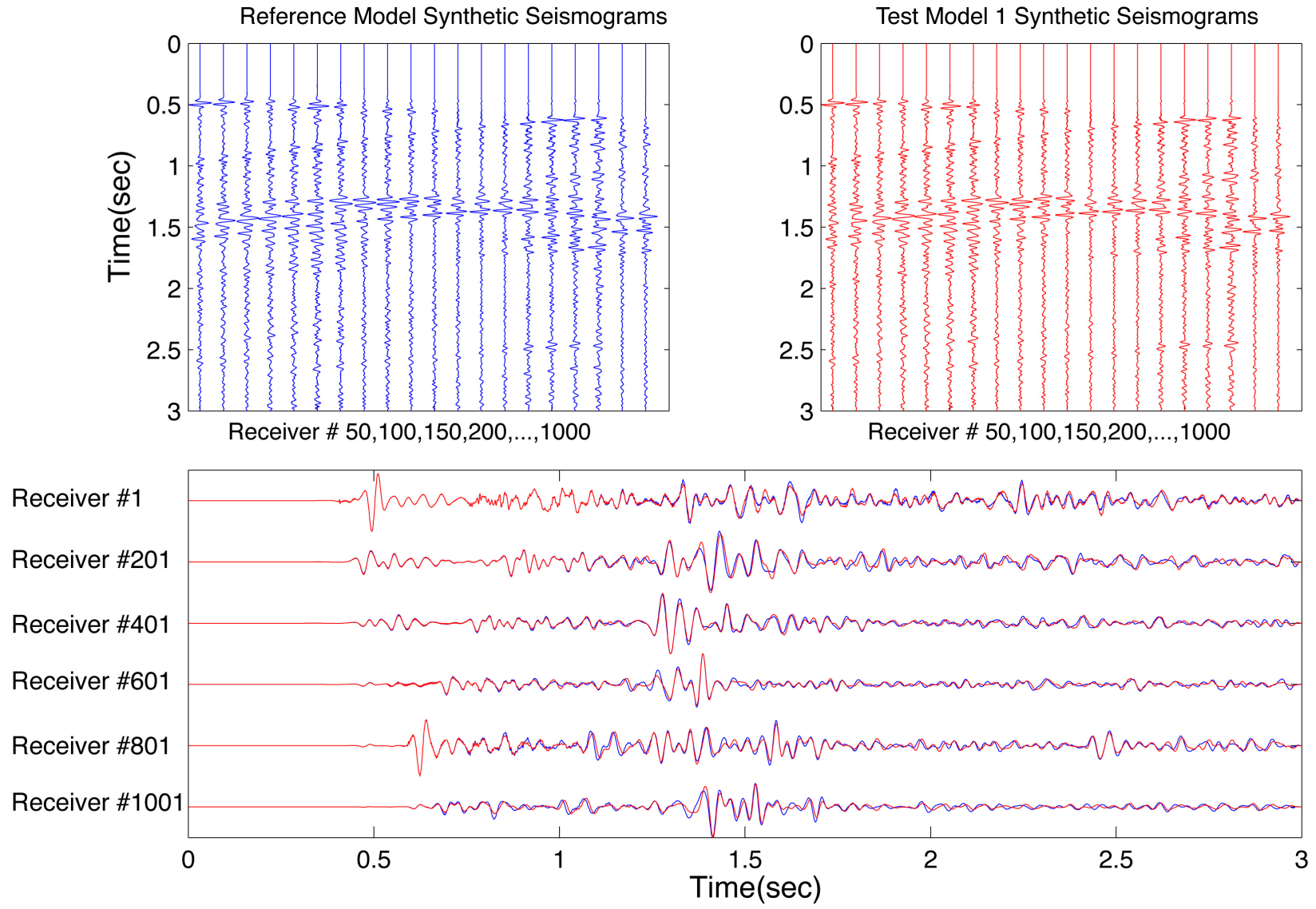

\section{Figure 2.}



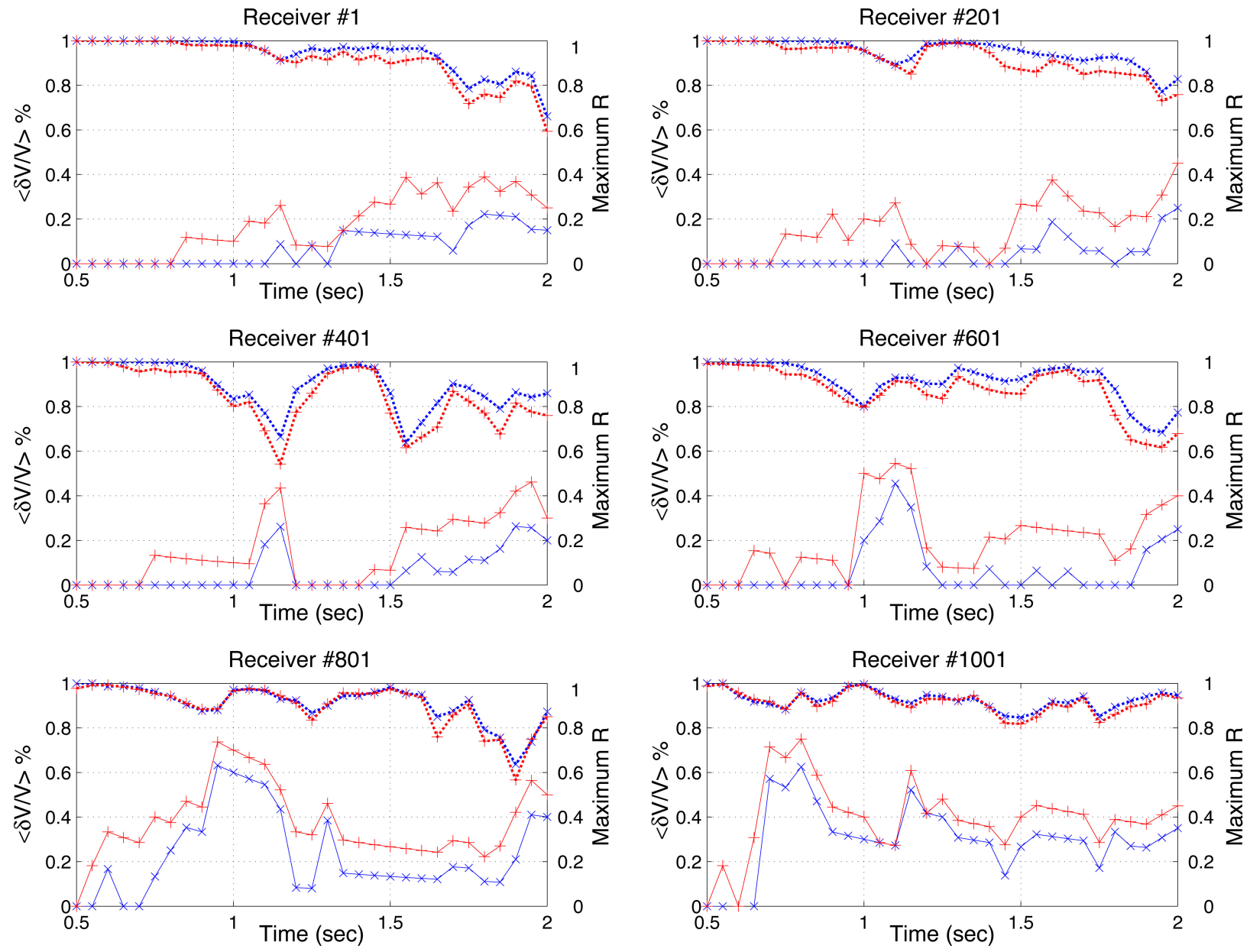

Figure 3. 


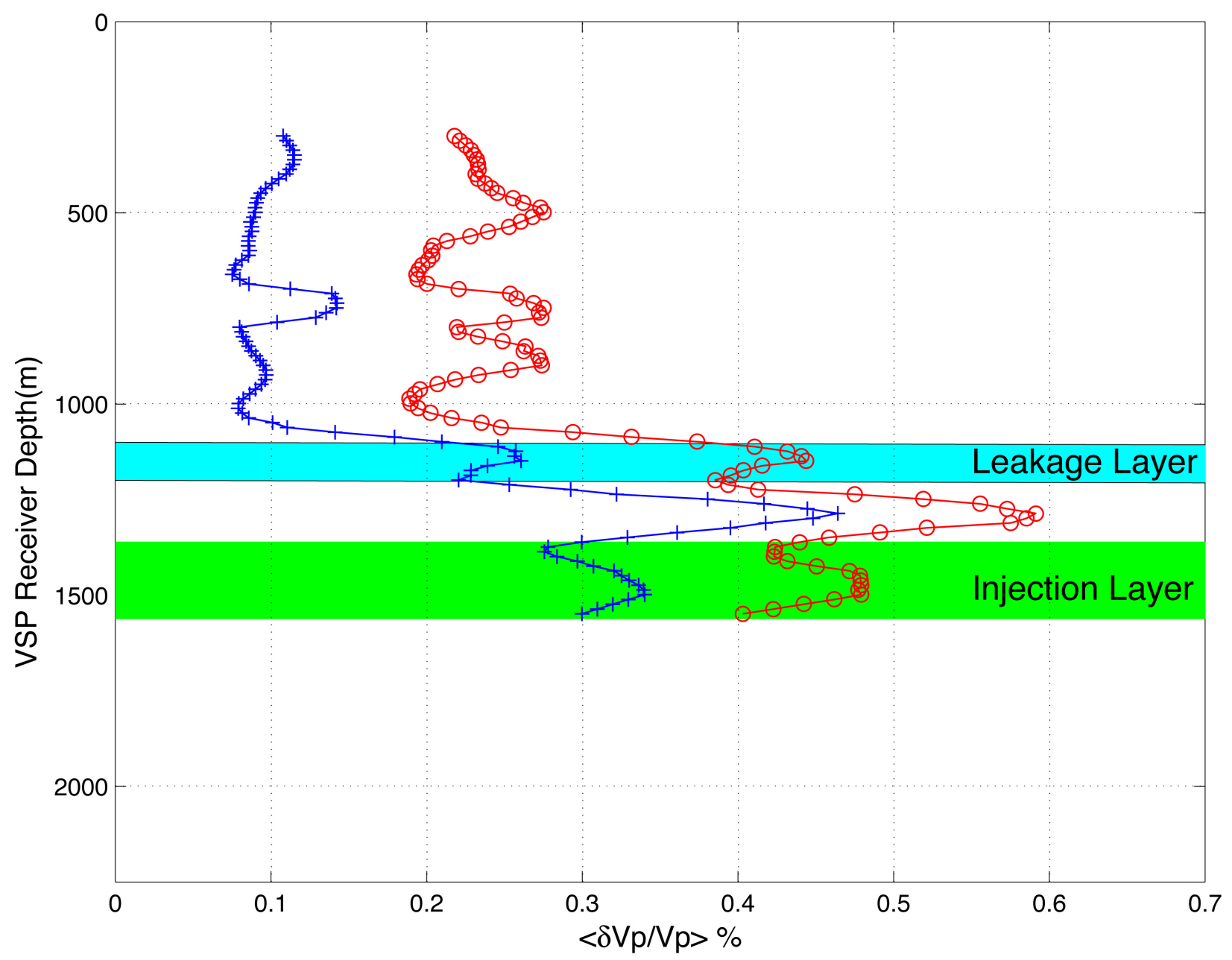

Figure 4. 


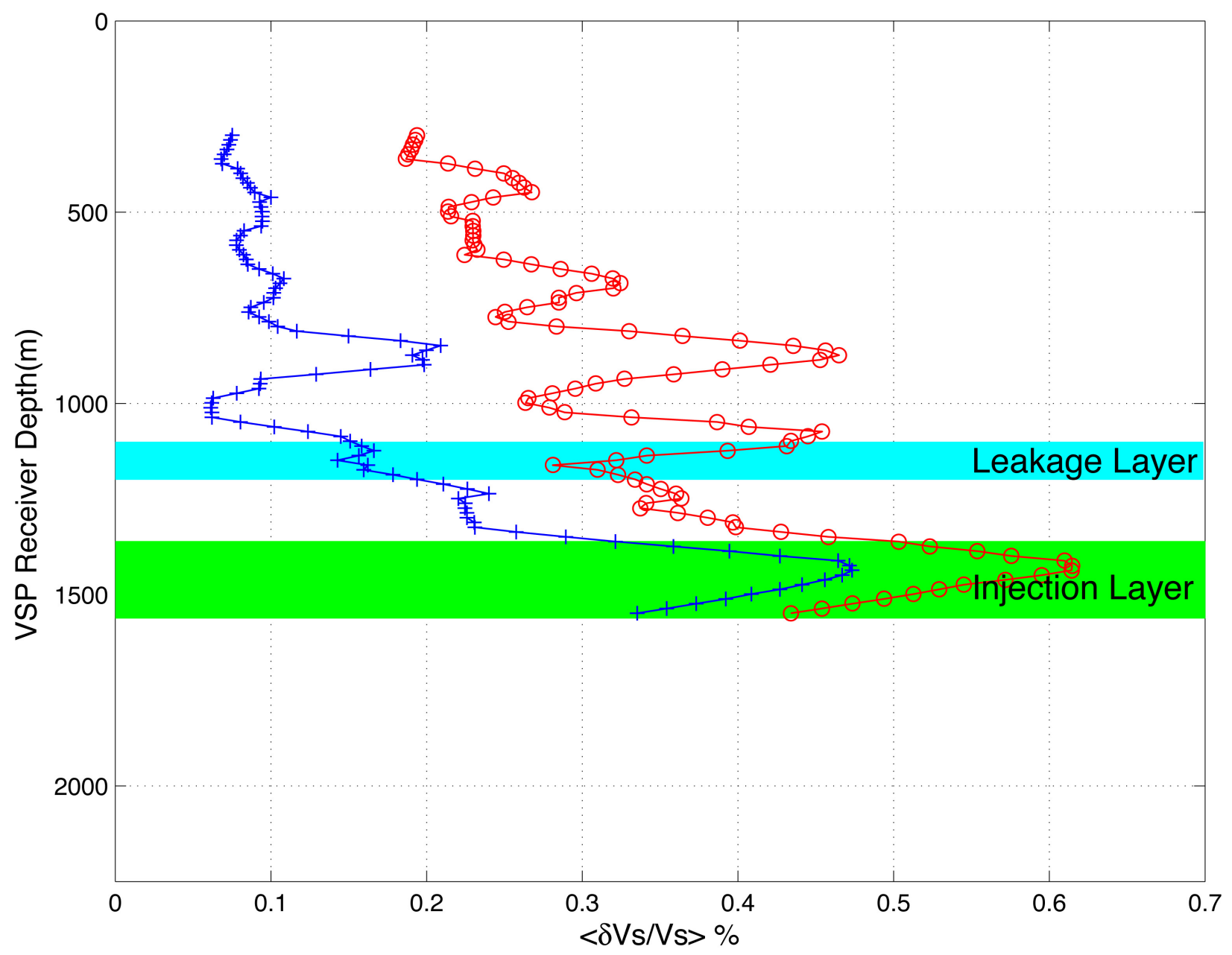

Figure 5. 

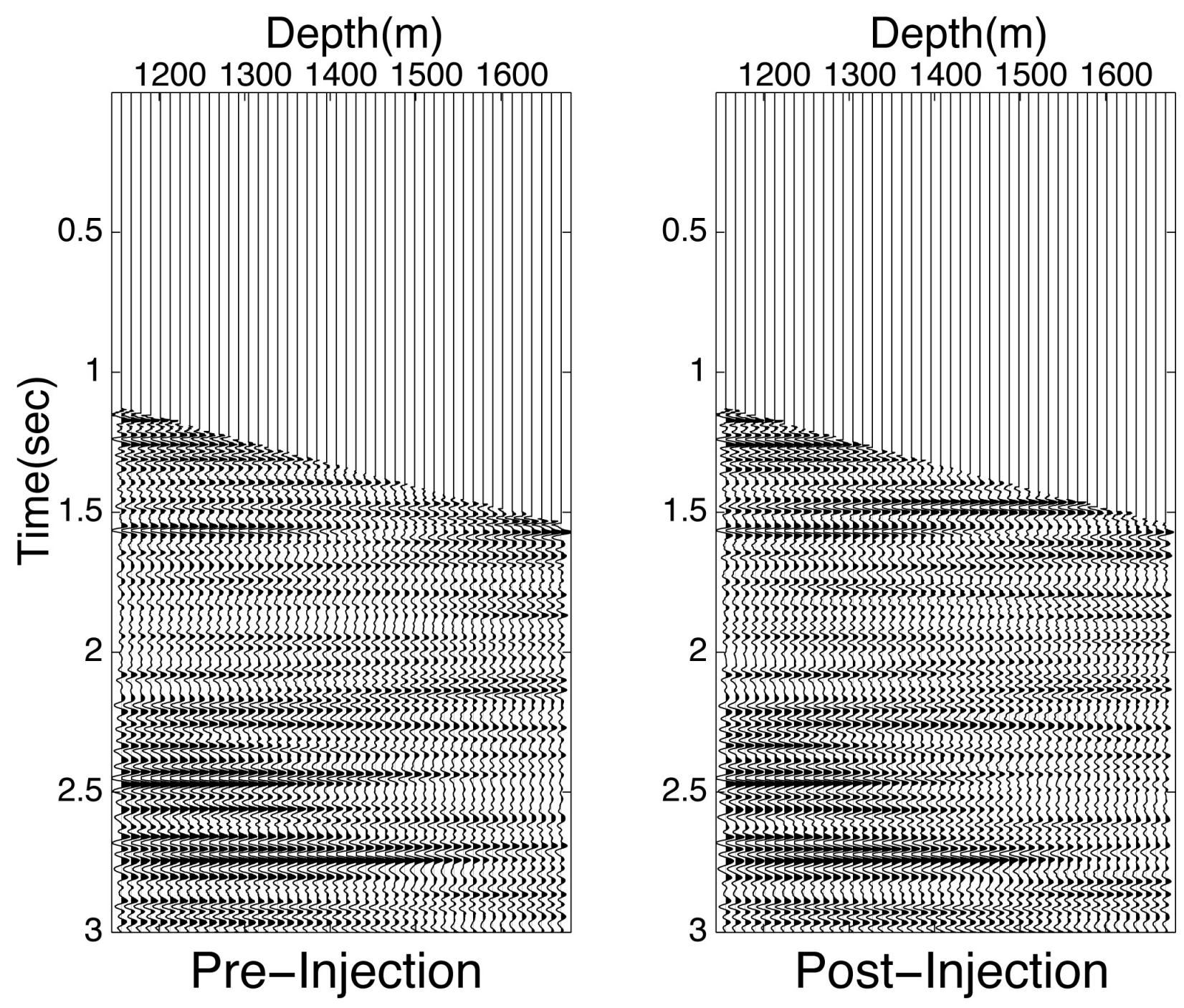

Figure 6. 


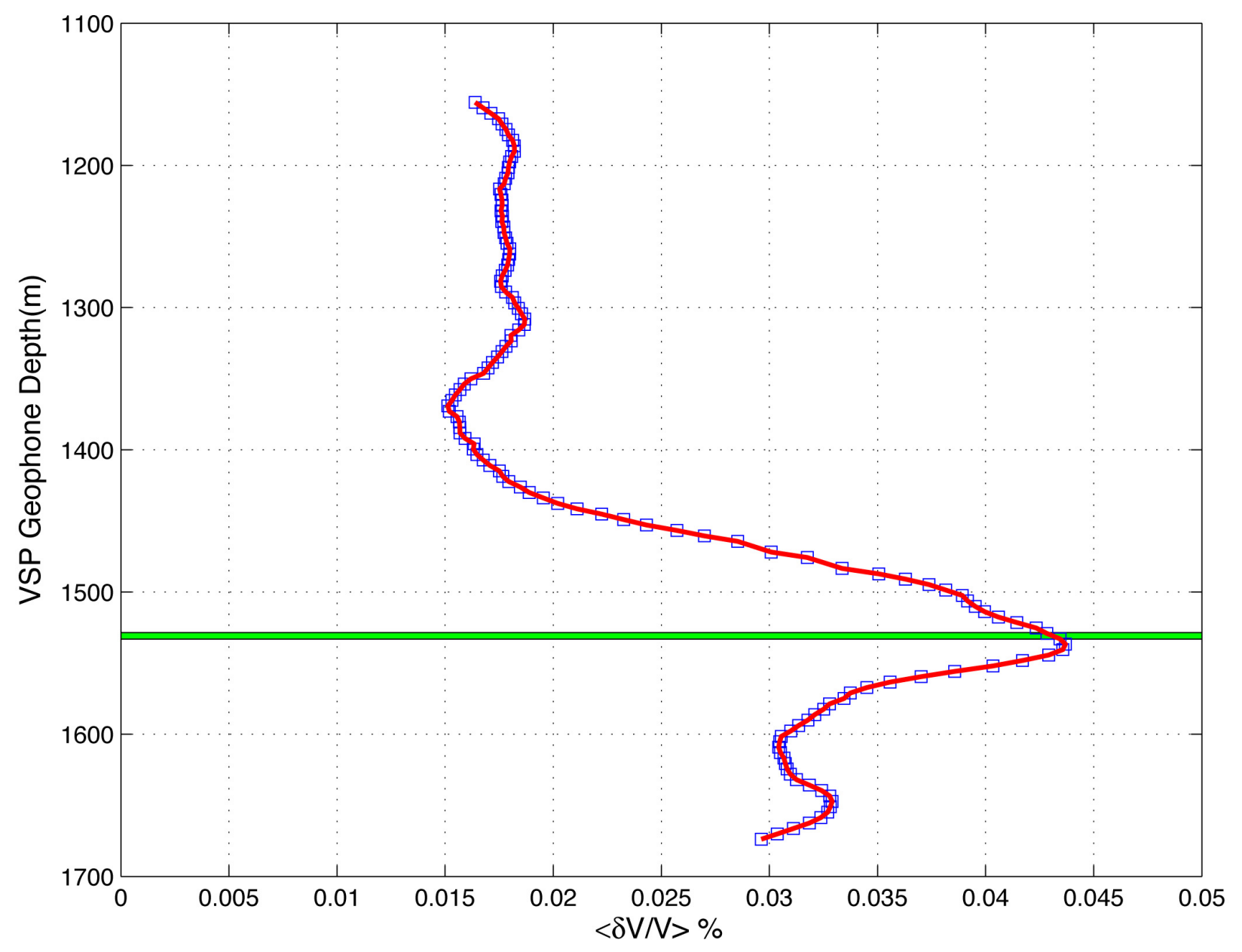

Figure 7. 

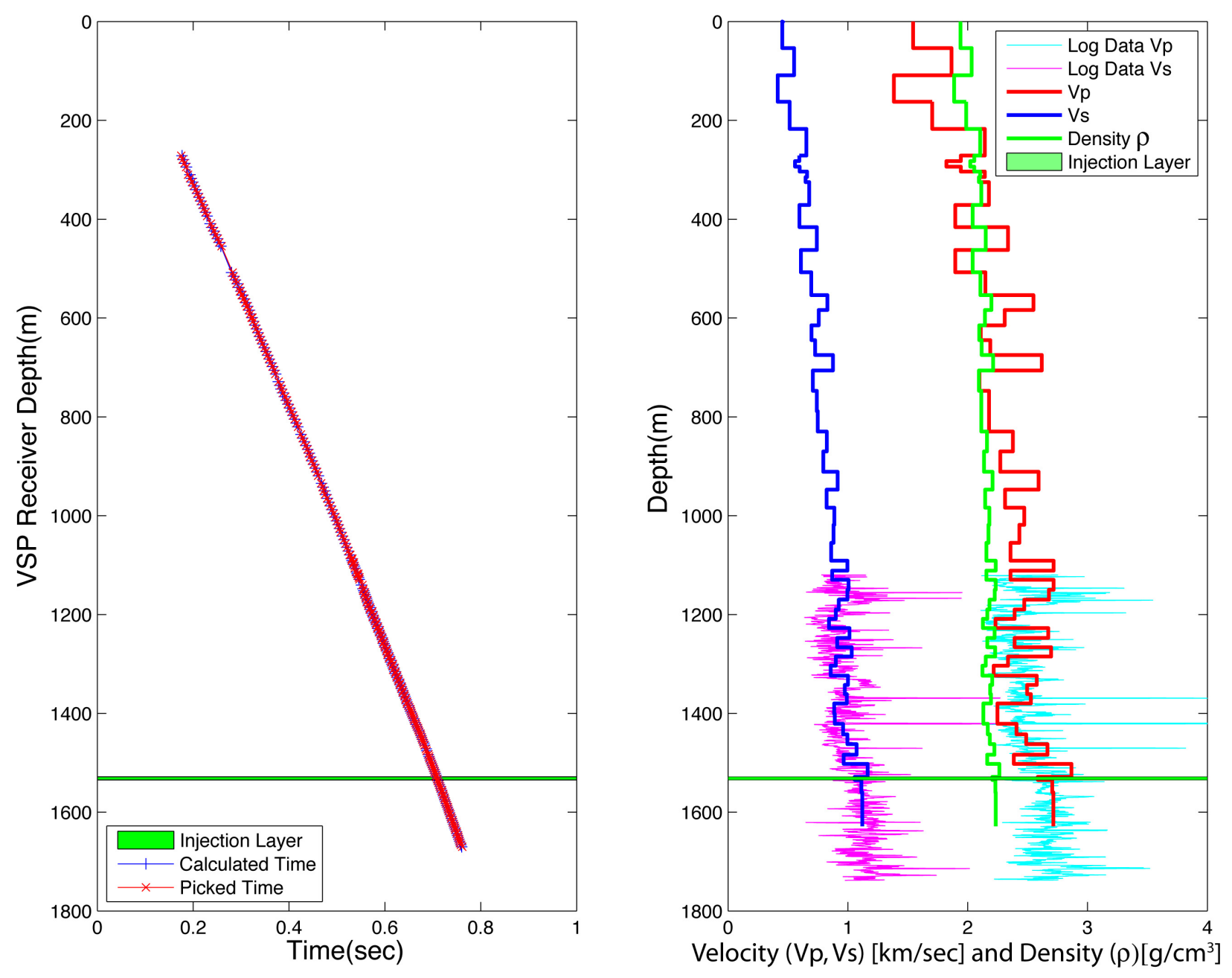

Figure 8. 


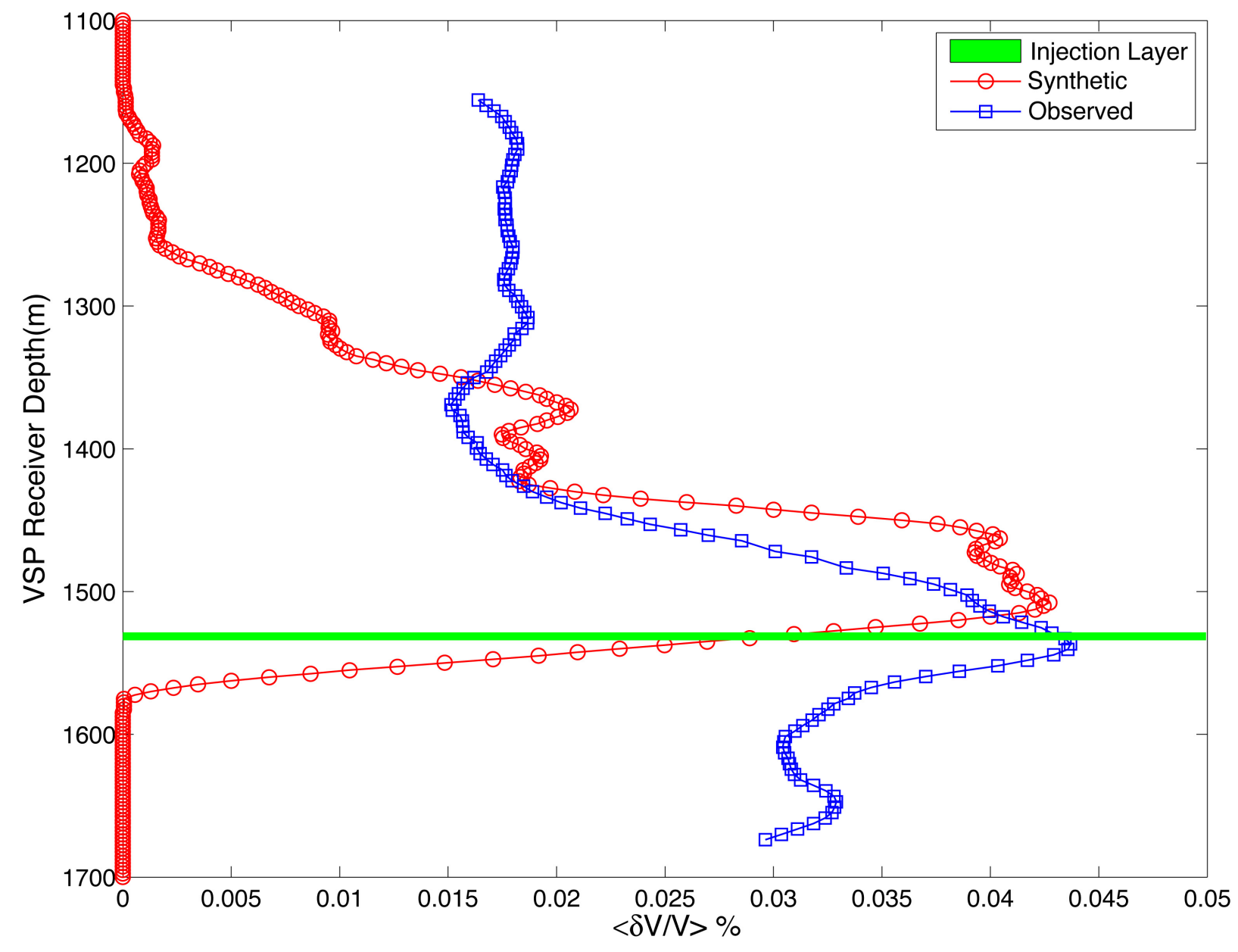

Figure 9. 